\title{
On the Hermite-Hadamard type inequalities
}

\author{
Chang-Jian Zhao ${ }^{1 *}$, Wing-Sum Cheung ${ }^{2}$ and Xiao-Yan Li ${ }^{3}$
}

\section{"Correspondence:}

chjzhao@163.com:

chjzhao@yahoo.com.cn

${ }^{1}$ Department of Mathematics, China

Jiliang University, Hangzhou,

310018, P.R. China

Full list of author information is

available at the end of the article

\begin{abstract}
In the present paper, we establish some new Hermite-Hadamard type inequalities involving two functions. Our results in a special case yield recent results on Hermite-Hadamard type inequalities.
\end{abstract}

MSC: $26 \mathrm{D} 15$

Keywords: Hermite-Hadamard inequality; Barnes-Godunova-Levin inequality; Minkowski integral inequality; Hölder inequality

\section{Introduction}

The following inequality is well known in the literature as Hermite-Hadamard's inequality [1].

Theorem 1.1 Let $f:[a, b] \subset \mathbb{R} \rightarrow \mathbb{R}$ be a convex function on an interval of real numbers. Then the following Hermite-Hadamard inequality for convex functions holds:

$$
f\left(\frac{a+b}{2}\right) \leq \frac{1}{b-a} \int_{a}^{b} f(x) d x \leq \frac{f(a)+f(b)}{2} .
$$

If the function $f$ is concave, the inequality (1.1) can be written as follows:

$$
f\left(\frac{a+b}{2}\right) \geq \frac{1}{b-a} \int_{a}^{b} f(x) d x \geq \frac{f(a)+f(b)}{2} .
$$

Recently, many generalizations, extensions and variants of this inequality have appeared in the literature (see, e.g., [2-10]) and the references given therein. In particular, in 2010, Özdemir and Dragomir [11] established some new Hermite-Hadamard inequalities and other integral inequalities involving two functions in $\mathbb{R}$. Following this work, the main purpose of the present paper is to establish some dual Hermite-Hadamard type inequalities involving two functions in $\mathbb{R}^{2}$. Our results provide some new estimates on such type of inequalities.

\section{Preliminaries}

A region $D \subset \mathbb{R}^{2}$ is called convex if it contains the close line segment joining any two of its points, or equivalently, if $\lambda x_{1}+(1-\lambda) x_{2}, \lambda y_{1}+(1-\lambda) y_{2} \in D$ whenever $x\left(x_{1}, y_{1}\right), y\left(x_{2}, y_{2}\right) \in D$ and $0 \leq \lambda \leq 1$.

(c) 2013 Zhao et al.; licensee Springer. This is an Open Access article distributed under the terms of the Creative Commons Attribution License (http://creativecommons.org/licenses/by/2.0), which permits unrestricted use, distribution, and reproduction in any medium, provided the original work is properly cited. 
Let $z=f(x, y)$ be a duality function on the convex region $D \subset \mathbb{R}^{2} . z=f(x, y)$ is called a duality convex function on the convex region $D$ if

$$
f\left[\lambda x_{1}+(1-\lambda) x_{2}, \lambda y_{1}+(1-\lambda) y_{2}\right] \leq \lambda f\left(x_{1}, y_{1}\right)+(1-\lambda) f\left(x_{2}, y_{2}\right),
$$

whenever $\left(x_{1}, y_{1}\right),\left(x_{2}, y_{2}\right) \in D$ and $0 \leq \lambda \leq 1$.

If the function $f(x, y)$ is concave, the inequality (2.1) can be written as follows:

$$
f\left[\lambda x_{1}+(1-\lambda) x_{2}, \lambda y_{1}+(1-\lambda) y_{2}\right] \geq \lambda f\left(x_{1}, y_{1}\right)+(1-\lambda) f\left(x_{2}, y_{2}\right) .
$$

Let $x=\left(x_{11}, \ldots, x_{1 n}, \ldots, x_{m 1}, \ldots, x_{m n}\right)$ and $p=\left(p_{11}, \ldots, p_{1 n}, \ldots, p_{m 1}, \ldots, p_{m n}\right)$ be two positive $n m$-tuples, and let $r \in \mathbb{R} \cup\{+\infty,-\infty\}$. Then, on putting $P_{m n}=\sum_{k_{2}=1}^{n} \sum_{k_{1}=1}^{m} p_{k_{1} k_{2}}$, it easy follows that if $-\infty \leq r<s \leq+\infty$, then

$$
M_{m n}^{[r]} \leq M_{m n}^{[s]}
$$

(also see, e.g., [1, p.15]). Here, the $r$ th power mean of $x$ with weights $p$ is the following: $M_{m n}^{[r]}=\left(\frac{1}{P_{m n}} \sum_{k_{2}=1}^{n} \sum_{k_{1}=1}^{m} p_{k_{1} k_{2}} x_{k_{1} k_{2}}^{r}\right)^{1 / r}$ if $r \neq+\infty, 0,-\infty ; M_{m n}^{[r]}=\left(\prod_{k_{2}=1}^{n} \prod_{k_{1}=1}^{m} x_{k_{1} k_{2}}^{p_{k_{1}} k_{2}}\right)^{P_{m n}}$ if $r=0 ; M_{m n}^{[r]}=\min \left(x_{11}, \ldots, x_{1 n}, \ldots, x_{n 1}, \ldots, x_{m n}\right)$ if $r=-\infty$ and $M_{m n}^{[r]}=\max \left(x_{11}, \ldots, x_{1 n}, \ldots, x_{n 1}\right.$, $\left.\ldots, x_{m n}\right)$ if $r=+\infty$.

Let $f(x, y):[a, b] \times[c, d] \rightarrow \mathbb{R}$, and $p \geq 1$. Now, we define the $p$-norm of the function $f(x, y)$ on $[a, b] \times[c, d]$ as follows:

$$
\|f(x, y)\|_{p}=\left(\int_{a}^{b} \int_{c}^{d}|f(x, y)|^{p} d x d y\right)^{1 / p}, \quad 1 \leq p<\infty,
$$

and

$$
\|f(x, y)\|_{p}=\sup |f(x, y)|, \quad p=\infty,
$$

and $L^{p}([a, b] \times[c, d])$ is the set of all functions $f(x, y):[a, b] \times[c, d] \rightarrow \mathbb{R}$ such that $\|f(x, y)\|_{p}<\infty$.

Lemma 2.1 (see [12]) (Barnes-Godunova-Levin inequality) Let $f(x, y), g(x, y)$ be nonnegative concave functions on $[a, b] \times[c, d]$, then for $p, q>1$ we have

$$
\|f(x, y)\|_{p}\|g(x, y)\|_{q} \leq B(p, q) \int_{a}^{b} \int_{c}^{d} f(x, y) g(x, y) d x d y,
$$

where

$$
B(p, q)=\frac{6[(b-a)(d-c)]^{1 / p+1 / q-1}}{(p+1)^{1 / p}(q+1)^{1 / q}} .
$$

Lemma 2.2 (see [1]) (Hermite-Hadamard inequality) Let $f(x, y):[a, b] \times[c, d] \subset \mathbb{R}^{2} \rightarrow \mathbb{R}$ be a convex function. Then the following dual Hermite-Hadamard inequality for convex functions holds:

$$
f\left(\frac{a+c}{2}, \frac{b+d}{2}\right) \leq \frac{1}{(b-a)(d-c)} \int_{a}^{b} \int_{c}^{d} f(x, y) d x d y \leq \frac{f(a, b)+f(c, d)}{2} .
$$


The inequality is reversed if the function $f(x, y)$ is concave.

Lemma 2.3 (see [13]) (A reversed Minkowski integral inequality) Let $f(x, y)$ and $g(x, y)$ be positive functions satisfying

$$
0<m \leq \frac{f(x, y)}{g(x, y)} \leq M, \quad(x, y) \in[a, b] \times[c, d] .
$$

Then

$$
\|f(x, y)\|_{p}+\|g(x, y)\|_{p} \leq c\|f(x, y)+g(x, y)\|_{p}
$$

where $c=[M(m+1)+(M+1)] /[(m+1)(M+1)]$.

\section{Main results}

Our main results are established in the following theorems.

Theorem 3.1 Let $p, q>1$ and let $f(x, y), g(x, y):[a, b] \times[c, d] \rightarrow \mathbb{R}$ be nonnegative functions such that $f(x, y)^{p}$ and $g(x, y)^{q}$ are concave on $[a, b] \times[c, d]$. Then

$$
\begin{aligned}
& \frac{f(a, b)+f(c, d)}{2} \times \frac{g(a, b)+g(c, d)}{2} \\
& \quad \leq \frac{1}{[(b-a)(d-c)]^{1 / p+1 / q}} B(p, q) \int_{a}^{b} \int_{c}^{d} f(x, y) g(x, y) d x d y,
\end{aligned}
$$

where $B(p, q)$ is the Barnes-Godunova-Levin constant given by (2.4).

Proof Observe that whenever $f^{p}(x, y)$ is concave on $[a, b] \times[c, d]$, the nonnegative function $f(x, y)$ is also concave on $[a, b] \times[c, d]$. Namely,

$$
f[\lambda a+(1-\lambda) c, \lambda b+(1-\lambda) d]^{p} \geq \lambda f(a, b)^{p}+(1-\lambda) f(c, d)^{p},
$$

that is,

$$
f[\lambda a+(1-\lambda) c, \lambda b+(1-\lambda) d] \geq\left(\left(\lambda f(a, b)^{p}+(1-\lambda) f(c, d)^{p}\right)\right)^{1 / p},
$$

and $p>1$, using the power-mean inequality (2.3), we obtain

$$
f[\lambda a+(1-\lambda) c, \lambda b+(1-\lambda) d] \geq \lambda f(a, b)+(1-\lambda) f(c, d) .
$$

For $q>1$, similarly, if $g^{q}(x, y)$ is concave on $[a, b] \times[c, d]$, the nonnegative function $g(x, y)$ is concave on $[a, b] \times[c, d]$.

In view that $f^{p}(x, y)$ and $g^{q}(x, y)$ are concave functions on $[a, b] \times[c, d]$, from Lemma 2.2, we get

$$
\begin{aligned}
\left(\frac{f(a, b)^{p}+f(c, d)^{p}}{2}\right)^{1 / p} & \leq \frac{1}{[(b-a)(d-c)]^{1 / p}}\left(\int_{a}^{b} \int_{c}^{d} f(x, y)^{p} d x d y\right)^{1 / p} \\
& \leq f\left(\frac{a+c}{2}, \frac{b+d}{2}\right)
\end{aligned}
$$


and

$$
\begin{aligned}
\left(\frac{g(a, b)^{p}+g(c, d)^{q}}{2}\right)^{1 / q} & \leq \frac{1}{[(b-a)(d-c)]^{1 / q}}\left(\int_{a}^{b} \int_{c}^{d} g(x, y)^{q} d x d y\right)^{1 / q} \\
& \leq g\left(\frac{a+c}{2}, \frac{b+d}{2}\right)
\end{aligned}
$$

By multiplying the above inequalities, we obtain

$$
\begin{aligned}
& \left(\frac{f(a, b)^{p}+f(c, d)^{p}}{2}\right)^{1 / p}\left(\frac{g(a, b)^{p}+g(c, d)^{q}}{2}\right)^{1 / q} \\
& \quad \leq \frac{1}{[(b-a)(d-c)]^{1 / p+1 / q}}\left(\int_{a}^{b} \int_{c}^{d} f(x, y)^{p} d x d y\right)^{1 / p}\left(\int_{a}^{b} \int_{c}^{d} g(x, y)^{q} d x d y\right)^{1 / q}
\end{aligned}
$$

If $p, q>1$, then it is easy to show that

$$
\left(\frac{f(a, b)^{p}+f(c, d)^{p}}{2}\right)^{1 / p} \geq \frac{f(a, b)+f(c, d)}{2}
$$

and

$$
\left(\frac{g(a, b)^{q}+g(c, d)^{q}}{2}\right)^{1 / q} \geq \frac{g(a, b)+g(c, d)}{2}
$$

Thus, by applying Barnes-Godunova-Levin inequality to the right-hand side of (3.4) with (3.5), (3.6), we get (3.1).

The proof is complete.

Remark 3.1 By multiplying inequalities (3.2), (3.3), we obtain

$$
\begin{aligned}
& \frac{1}{[(b-a)(d-c)]^{1 / p+1 / q}}\left(\int_{a}^{b} \int_{c}^{d} f(x, y)^{p} d x d y\right)^{1 / p}\left(\int_{a}^{b} \int_{c}^{d} g(x, y)^{q} d x d y\right)^{1 / q} \\
& \quad \leq f\left(\frac{a+c}{2}, \frac{b+d}{2}\right) g\left(\frac{a+c}{2}, \frac{b+d}{2}\right) .
\end{aligned}
$$

By applying the Hölder inequality to the left-hand side of $(3.7)$ with $(1 / p)+(1 / q)=1$, we get

$$
\frac{1}{(b-a)(d-c)} \int_{a}^{b} \int_{c}^{d} f(x, y) g(x, y) d x d y \leq f\left(\frac{a+c}{2}, \frac{b+d}{2}\right) g\left(\frac{a+c}{2}, \frac{b+d}{2}\right) .
$$

Remark 3.2 Let $f(x, y)$ and $g(x, y)$ change to $f(x)$ and $g(x)$, respectively, and with suitable changes in Theorem 3.1 and Remark 3.1, we have the following.

Corollary 3.1 Let $p, q>1$ and let $f(x), g(x):[a, b] \rightarrow \mathbb{R}, a<b$, be nonnegative functions such that $f(x)^{p}$ and $g(x)^{q}$ are concave on $[a, b]$. Then

$$
\frac{f(a)+f(b)}{2} \cdot \frac{g(a)+g(b)}{2} \leq \frac{1}{(b-a)^{1 / p+1 / q}} B(p, q) \int_{a}^{b} f(x) g(x) d x
$$


and if $(1 / p)+(1 / q)=1$, then one has

$$
\frac{1}{b-a} \int_{a}^{b} f(x) g(x) d x \leq f\left(\frac{a+b}{2}\right) g\left(\frac{a+b}{2}\right) .
$$

This is just Theorem 2.1 established by Özdemir and Dragomir [11].

Theorem 3.2 Let $p \geq 1$ and let $\int_{a}^{b} \int_{c}^{d} f(x, y)^{p} d x d y<\infty$ and $\int_{a}^{b} \int_{c}^{d} g(x, y)^{p} d x d y<\infty$, and let $f(x, y), g(x, y):[a, b] \times[c, d] \rightarrow \mathbb{R}$ be positive functions with

$$
0<m \leq \frac{f(x, y)}{g(x, y)} \leq M, \quad \forall(x, y) \in[a, b] \times[c, d]
$$

Then

$$
\|f(x, y)\|_{p}^{2}+\|g(x, y)\|_{p}^{2} \geq\left(\frac{(M+1)(m+1)}{M}-2\right)\|f(x, y)\|_{p}\|g(x, y)\|_{p}
$$

Proof Since $f(x, y), g(x, y)$ are positive, as in the proof of Lemma 2.3 (see [13, p.2]), we have

$$
\left(\int_{a}^{b} \int_{c}^{d} f(x, y)^{p} d x d y\right)^{1 / p} \leq \frac{M}{M+1}\left(\int_{a}^{b} \int_{c}^{d}(f(x, y)+g(x, y))^{p} d x d y\right)^{1 / p}
$$

and

$$
\left(\int_{a}^{b} \int_{c}^{d} g(x, y)^{p} d x d y\right)^{1 / p} \leq \frac{1}{m+1}\left(\int_{a}^{b} \int_{c}^{d}(f(x, y)+g(x, y))^{p} d x d y\right)^{1 / p} .
$$

By multiplying the above inequalities and in view of the Minkowski inequality, we get

$$
\begin{aligned}
& \left(\int_{a}^{b} \int_{c}^{d} f(x, y)^{p} d x d y\right)^{1 / p}\left(\int_{a}^{b} \int_{c}^{d} g(x, y)^{p} d x d y\right)^{1 / p} \\
& \leq \leq \frac{M}{(M+1)(m+1)}\left(\int_{a}^{b} \int_{c}^{d}(f(x, y)+g(x, y))^{p} d x d y\right)^{2 / p} \\
& \leq \frac{M}{(M+1)(m+1)}\left(\left(\int_{a}^{b} \int_{c}^{d} f(x, y)^{p} d x d y\right)^{1 / p}\right. \\
& \left.\quad+\left(\int_{a}^{b} \int_{c}^{d} g(x, y)^{p} d x d y\right)^{1 / p}\right)^{2}
\end{aligned}
$$

Hence

$$
\begin{aligned}
& \left(\int_{a}^{b} \int_{c}^{d} f(x, y)^{p} d x d y\right)^{2 / p}+\left(\int_{a}^{b} \int_{c}^{d} g(x, y)^{p} d x d y\right)^{2 / p} \\
& \geq\left(\frac{(M+1)(m+1)}{M}-2\right)\left(\int_{a}^{b} \int_{c}^{d} f(x, y)^{p} d x d y\right)^{1 / p}\left(\int_{a}^{b} \int_{c}^{d} g(x, y)^{p} d x d y\right)^{1 / p} .
\end{aligned}
$$

This proof is complete.

Remark 3.3 Let $f(x, y)$ and $g(x, y)$ change to $f(x)$ and $g(x)$, respectively, and with suitable changes in (3.9), (3.9) reduces to an inequality established by Özdemir and Dragomir [11]. 
Theorem 3.3 If $f^{p}(x, y)$ and $g^{q}(x, y)$ are as in Theorem 3.1, then the following inequality holds:

$$
\frac{1}{(b-a)(d-c)}\|f(x, y)\|_{p}^{p} \cdot\|g(x, y)\|_{q}^{q} \geq \frac{(f(a, b)+f(c, d))^{p}(g(a, b)+g(c, d))^{q}}{2^{p+q}} .
$$

Proof If $f^{p}(x, y)$ and $g^{q}(x, y)$ are concave on $[a, b] \times[c, d]$, then from Lemma 2.2, we get

$$
\frac{f(a, b)^{p}+f(c, d)^{p}}{2} \leq \frac{1}{(b-a)(d-c)} \int_{a}^{b} \int_{c}^{d} f(x, y)^{p} d x d y
$$

and

$$
\frac{g(a, b)^{q}+g(c, d)^{q}}{2} \leq \frac{1}{(b-a)(d-c)} \int_{a}^{b} \int_{c}^{d} g(x, y)^{q} d x d y
$$

which imply that

$$
\begin{aligned}
& \frac{\left[f(a, b)^{p}+f(c, d)^{p}\right]\left[g(a, b)^{q}+g(c, d)^{q}\right]}{4} \\
& \quad \leq \frac{1}{[(b-a)(d-c)]^{2}} \int_{a}^{b} \int_{c}^{d} f(x, y)^{p} d x d y \int_{a}^{b} \int_{c}^{d} g(x, y)^{q} d x d y .
\end{aligned}
$$

On the other hand, if $p, q \geq 1$, from (2.3) we get

$$
\frac{f(a, b)^{p}+f(c, d)^{p}}{2} \leq 2^{-p}[f(a, b)+f(c, d)]^{p}
$$

and

$$
\frac{g(a, b)^{q}+g(c, d)^{q}}{2} \leq 2^{-q}[g(a, b)+g(c, d)]^{q},
$$

which imply that

$$
\begin{aligned}
& \frac{\left[f(a, b)^{p}+f(c, d)^{p}\right]\left[g(a, b)^{p}+g(c, d)^{q}\right]}{4} \\
& \quad \geq 2^{-p-q}[f(a, b)+f(c, d)]^{p}[g(a, b)+g(c, d)]^{q} .
\end{aligned}
$$

Combining (3.12) and (3.13), we obtain the desired inequality as

$$
\begin{aligned}
& 2^{-p-q}[f(a, b)+f(c, d)]^{p}[g(a, b)+g(c, d)]^{q} \\
& \quad \leq \frac{1}{[(b-a)(d-c)]^{2}}\|f(x, y)\|_{p}^{p} \cdot\|g(x, y)\|_{q}^{q} .
\end{aligned}
$$

This proof is complete.

Remark 3.4 Let $f(x, y)$ and $g(x, y)$ change to $f(x)$ and $g(x)$, respectively, and with suitable changes in (3.11), (3.11) reduces to an inequality established by Özdemir and Dragomir [11]. 
Theorem 3.4 Let $f(x, y), g(x, y):[a, b] \times[c, d] \rightarrow \mathbb{R}^{+}$be functions such that $f(x, y)^{p}, g(x, y)^{q}$ and $f(x, y) g(x, y)$ are in $L_{1}([a, b] \times[c, d])$, and

$$
0<m \leq \frac{f(x, y)}{g(x, y)} \leq M, \quad \forall(x, y) \in[a, b] \times[c, d], a, b, c, d \in[0, \infty) .
$$

Then

$$
\begin{aligned}
& \int_{a}^{b} \int_{c}^{d} f(x, y) g(x, y) d x d y \\
& \quad \leq c_{1}\left(\frac{\|f(x, y)\|_{p}^{p}+\|g(x, y)\|_{p}^{p}}{2}\right)+c_{2}\left(\frac{\|f(x, y)\|_{q}^{q}+\|g(x, y)\|_{q}^{q}}{2}\right),
\end{aligned}
$$

where

$$
c_{1}=\frac{2^{p}}{p}\left(\frac{M}{M+1}\right)^{p}, \quad c_{2}=\frac{2^{q}}{q}\left(\frac{1}{m+1}\right)^{q},
$$

and $(1 / p)+(1 / q)=1$ with $p>1$.

Proof Since $0<m \leq \frac{f(x, y)}{g(x, y)} \leq M, \forall(x, y) \in[a, b] \times[c, d]$, we have

$$
f(x, y) \leq \frac{M}{M+1}(f(x, y)+g(x, y))
$$

and

$$
g(x, y) \leq \frac{1}{m+1}(f(x, y)+g(x, y))
$$

In view of the Young-type inequality and using the elementary inequality

$$
(a+b)^{p} \leq 2^{p-1}\left(a^{p}+b^{p}\right), \quad p>1, a, b \in \mathbb{R}^{+},
$$

we have

$$
\begin{aligned}
\int_{a}^{b} \int_{c}^{d} f(x, y) g(x, y) d x d y \\
\leq \frac{1}{p}\left(\frac{M}{M+1}\right)^{p} \int_{a}^{b} \int_{c}^{d}(f(x, y)+g(x, y))^{p} d x d y \\
\quad+\frac{1}{q}\left(\frac{1}{m+1}\right)^{q} \int_{a}^{b} \int_{c}^{d}(f(x, y)+g(x, y))^{q} d x d y \\
\leq \frac{1}{p}\left(\frac{M}{M+1}\right)^{p} 2^{p-1} \int_{a}^{b} \int_{c}^{d}\left[f(x, y)^{p}+g(x, y)^{p}\right] d x d y \\
\quad+\frac{1}{q}\left(\frac{1}{m+1}\right)^{q} 2^{q-1} \int_{a}^{b} \int_{c}^{d}\left[f(x, y)^{q}+g(x, y)^{q}\right] d x d y .
\end{aligned}
$$

This completes the proof. 
Remark 3.5 Let $f(x, y)$ and $g(x, y)$ change to $f(x)$ and $g(x)$, respectively, and with suitable changes in (3.14), (3.14) reduces to an inequality established by Özdemir and Dragomir [11].

Competing interests

The authors declare that they have no competing interests.

\section{Authors' contributions}

C-JZ, W-SC and X-YL jointly contributed to the main results Theorems 3.1-3.4. All authors read and approved the final manuscript.

\section{Author details}

${ }^{1}$ Department of Mathematics, China Jiliang University, Hangzhou, 310018, P.R. China. ${ }^{2}$ Department of Mathematics, The University of Hong Kong, Pokfulam Road, Hong Kong, P.R. China. ${ }^{3}$ Department of Mathematics, Hunan Normal University, Changsha, 410000, P.R. China.

\section{Acknowledgements}

The first author's research is supported by Natural Science Foundation of China (10971205). The second author's research is partially supported by a HKU Seed Grant for Basic Research.

Received: 20 October 2012 Accepted: 18 April 2013 Published: 7 May 2013

\section{References}

1. Mitrinović, DS, Pecarič, JE, Fink, AM: Classical and New Inequalities in Analysis. Mathematics and Its Applications (East European Series), vol. 61. Kluwer Academic, Dordrecht (1993)

2. Kavurmaci, H, Avci, M, Özdemir, ME: New inequalities of Hermite-Hadamard type for convex functions with applications. J. Inequal. Appl. 2011, 86 (2011)

3. Özdemir, ME, Kavurmaci, H, Ocak Akdemir, A, Avci, M: Inequalities for convex and s-convex functions on $\Delta=[a, b] \times[c, d]$. J. Inequal. Appl. 2012, 20 (2012)

4. Dragomir, SS: A sequence of mappings associated with the Hermite-Hadamard inequalities and applications. Appl. Math. 49, 123-140 (2004)

5. Kotrys, D: Hermite-Hadamard inequality for convex stochastic processes. Aequ. Math. 83, 143-151 (2012)

6. Makó, J, Páles, Z: Hermite-Hadamard inequalities for generalized convex functions. Aequ. Math. 69, 32-40 (2005)

7. Ger, R, Pečarić, J: On vector Hermite-Hadamard differences controlled by their scalar counterparts. Int. Ser. Numer. Math. 161, 165-173 (2010)

8. Xi, B-Y, Bai, R-F, Qi, F: Hermite-Hadamard type inequalities for the $m$ - and $(\alpha, m)$-geometrically convex functions. Filomat 27, 1-7 (2013)

9. Dragomir, SS, Hunt, E, Pearce, CEM: Interpolating maps, the modulus map and Hadamard's inequality. In: Optimization, Part 1. Springer Optimization and Its Applications, vol. 32, pp. 207-223 (2009)

10. Niculescu, CP: The Hermite-Hadamard inequality for convex functions of a vector variable. Math. Inequal. Appl. 5(4), 619-623 (2002)

11. Set, E, Özdemir, ME, Dragomir, SS: On the Hermite-Hadamard inequality and other integral inequalities involving two functions. J. Inequal. Appl. 2010, Article ID 148102 (2010)

12. Pachpatte, BG: Inequalities for Differentiable and Integral Equations. Academic Press, Boston (1997)

13. Bougoffa, L: On Minkowski and Hardy integral inequalities. J. Inequal. Pure Appl. Math. 7(2), Article ID 60 (2006)

doi:10.1186/1029-242X-2013-228

Cite this article as: Zhao et al.: On the Hermite-Hadamard type inequalities. Journal of Inequalities and Applications $20132013: 228$

\section{Submit your manuscript to a SpringerOpen ${ }^{\ominus}$ journal and benefit from:}

- Convenient online submission

- Rigorous peer review

- Immediate publication on acceptance

- Open access: articles freely available online

- High visibility within the field

- Retaining the copyright to your article 